\title{
Interferometer Observations of Extragalactic Radio Sources at Decameter Wavelengths
}

\author{
A.V. Megn, S.Ya. Braude, S.L. Rashkovsky, V.A. Shepelev, \\ N.K.Sharykin and G.A. Inyutin \\ Institute of Radio Astronomy NAS Ukraine, 4 Chervonopraporna str., \\ Kharkiv, 61002, Ukraine
}

\begin{abstract}
The angular structure of extragalactic radio sources was studied at decameter wavelengths with resolution up to 1 arcsec using the URAN interferometer network. A extended low frequency halo with steep spectrum is detected for some of them and the known components of others become, as a rule, bigger at these wavelengths.
\end{abstract}

The progress in modern astronomy is strongly connected with investigating the fine structure of cosmic radio sources, and the studies are carried out in a wide frequency range. However, very low frequency investigations were hampered for long because of problems of high levels of interference, high temperature of the galactic background, strong influence of the ionosphere and difficulties in construction of instruments with a high resolving power.

The low frequency VLBI network URAN operating the range of 16 to 25 $\mathrm{MHz}$ was designed in Ukraine to fill the gap (Megn et al. 1997). The network consists of 5 radio telescopes making up 4 interferometers with the baselines 42 to $913 \mathrm{~km}$ with UTR-2 radio telescope operating as the main antenna of the interferometers. The network angular resolution reaches $1 \operatorname{arcsec}$ at the highest frequency of the range, and its sensitivity is about $20 \mathrm{Jy}$.

Regular observations of extragalactic radio sources are carried out with the URAN interferometer network at decameter wavelengths for a few years. Some results of the studies are presented in the paper.

The angular structures of quasars change as compared with the images obtained at higher frequencies: the most compact details disappear due to reabsorbtion and extended components with steep spectra appear. These latter cannot be observed at higher frequencies and their diameters often exceed the full size of the source measured at higher frequencies. As a rule the extended components make a major contribution to the decameter emission of the source. Most of the observed quasars possess a "core-halo" type structure. For example, at decameter wavelengths, the brightness distribution of quasar 3C296 consists of two components with half power angular size of about $26^{\prime \prime}$ and $2^{\prime \prime}$ with their separation less than $5^{\prime \prime}($ Megn et al. 1990). At the same time the source full size at the frequency $408 \mathrm{MHz}$ does not exceed $10^{\prime \prime}$. The quasars 3C154 (Megn et al. 1998) and 3C254 (Megn et al. 1996) have the same angular structure at decameter wavelengths. On the contrary, the most probable low frequency model of the quasar 3C47 consists of two separated components. The positions of these components coincide with the lobes observed in the higher frequency 
maps, but their diameters are larger than the high frequency sizes (Megn et al. 1995).

The radio galaxies at low frequencies, as a rule, have a classical double structure, while their extended lobes become essentially bigger than the higher frequency components. Compact components, which are associated with optical galaxies, usually have flat spectra and often are not observed at decameter wavelengths. Such brightness distribution which is characteristic of galaxies, is shown by 3C111 (Megn et al. 1999) and 3C338, but the location of the extended lobes slightly differ from that in higher frequency maps. The radio source $3 \mathrm{C} 123$ has only one component and its diameter is equal to the higher frequency source size (Megn et al. 1987). The position of both components of the unidentified source 3C134 measured at low frequencies coincides with their location at higher frequency maps and their diameters exceed the size of the components measured at higher frequencies (Megn et al. 1985).

We obtained the following parameters of the studied sources: dimensions and flux densities of all components, their spectra down to very low frequencies, as well as reasons for spectral distortions at low frequencies and physical conditions in space plasma for some sources were clarified.

\section{References}

Megn, A.V, Braude, S.Ya., Rashkovsky, S.L, Falkovich, I.S., Sharykin, N.K., Shepelev, V.A., \& Khristenko, A.D. 1985, Astronomicheskii Zhurnal, 62, 38

Megn, A.V., Braude, S.Ya., Rashkovsky, S.L, Falkovich, I.S., Sharykin, N.K., Shepelev, V.A., Inyutin, G.A., \& Khristenko, A.D. 1987, Pisma v AZh, 13,751

Megn, A.V., Braude, S.Ya., Rashkovsky, S.L, Sharykin, N.K., Shepelev, V.A., Inyutin, G.A., \& Khristenko, A.D. 1990, Izvestia VUZov, Radiofizika, 33, 534

Megn, A.V., Braude, S.Ya., Rashkovsky, S.L, Sharykin, N.K., Shepelev, V.A., Inyutin, G.A., Khristenko, A.D, \& Podgorny, G.S. 1995, Izvestiya VUZov, Radiofizika, 38, 511

Megn, A.V., Braude, S.Ya., Rashkovsky, S.L, Sharykin, N.K., Shepelev, V.A., Inyutin, G.A., \& Podgorny, G.S. 1996, Kinematika i physika nebesnyh tel, 12,3

Megn, A.V., Braude, S.Ya., Rashkovsky, S.L, Sharykin, N.K., Shepelev, V.A., Inyutin, G.A., Bulatsen, V.G., Bratsenko, A.I., Koshovij, V.V., Romanchev, Yu.V., Thesevich, V.P., \& Galanin, V.V. 1997, Radiofisika i radioastronomiya, 2, 385

Megn, A.V., Braude, S.Ya., Rashkovsky, S.L, Sharykin, N.K., Shepelev, V.A., Inyutin, G.A., Khristenko, A.D., Brazhenko, A.I., \& Bulatsen, V.G. 1998, Astronomy Reports, 42,722

Megn, A.V., Braude, S.Ya., Rashkovsky, S.L, Sharykin, N.K., Shepelev, V.A., \& Inyutin, G.A. 1999, Astronomy Reports, 43, 428 